\title{
Duration of Viral Clearance in Children With SARS-CoV-2 Infection in Rajasthan, India
}

\author{
Manohar Lal Gupta, ${ }^{1}$ Sunil Gothwal, ${ }^{1}$ Raj Kumar Gupta, ${ }^{1}$ Ram Babu Sharma, ${ }^{1}$ Jeetam Singh Meena, ${ }^{1}$ \\ Pawan Kumar Sulaniya, ${ }^{1}$ Deveshwar Dev ${ }^{1}$ and Deepak Kumar Gupta ${ }^{2}$ \\ From Department of ${ }^{1}$ Pediatrics, SPMCH Institute, SMS Medical College, and ${ }^{2}$ Centre for Data Analysis, Research and Training \\ (CDART) Jaipur, Rajasthan, India.
}

Correspondence to: Prof RK Gupta, Department of Pediatric Medicine, SMS Medical College, Jaipur, India. rkguptadr@hotmail.com Received: September 18, 2020; Initial Review: September 21, 2020; Accepted: November 29, 2020
Objective: To study the clinical and laboratory profile and to assess period for viral clearance in COVID 19 children. Methods: We reviewed hospital records of children ( $<18$ years) admitted from 1 April to 31 May, 2020 at a tertiary-care public hospital and identified those positive for severe acute respiratory syndrome corona virus (SARS-CoV-2) by RT-PCR of respiratory secretions. Results: $81.2 \%$ of the 85 children studied were asymptomatic and $3(8.5 \%)$ died. Severe lymphopenia (43.8\%), raised C-reactive protein (93.8\%), raised erythrocyte sedimentation rate $(75 \%)$ and high $(>500 \mathrm{ng} / \mathrm{mL}$ ) levels of $\mathrm{D}$-dimer $(37.5 \%)$ were common. Median (IQR) duration of viral shedding was 7 (5-10) days, with range of 2 to 45 days; $96.3 \%$ had viral clearance within 14 days. Conclusions: Majority of children aged $<18$ years with SARS-CoV2 infection had viral clearance within 14 days.

Keywords: COVID-19, Management, Outcome, Viral shedding.

Published online: 29 November, 2020; PII:S097475591600255

$\mathrm{N}$ ovel corona virus disease 2019 (COVID-19), caused by severe acute respiratory syndrome corona virus 2 (SARS-CoV-2), has spread widely in India. Children account for $1-5 \%$ of diagnosed COVID-19 cases [1]. Clinical features, disease progression and outcome in children is milder compared to adults. To date, studies on COVID-19 in Indian children are scanty [2]. Moreover, there is limited literature over duration of viral clearance in COVID-19 children. We report profile of SARS-CoV-2 positive children and their viral clearance pattern at a public hospital in northern India.

\section{METHODS}

This study was hospital-based record review conducted in a tertiary care center attached to a government medical college. Data of all children (aged up to 18 years) positive for SARS-CoV-2 by RT-PCR of respiratory secretions (nasopharyngeal/oropharyngeal/nasal swab), admitted in COVID-19 ward of the hospital, from 1 April, 2020 to 31 May, 2020 were included in study. All children coming to COVID-19 OPD/ Influenza like illness OPD and suspected for COVID-19 according to ICMR guidelines, were tested by RT-PCR of respiratory secretions [3].

Nasopharyngeal or oropharyngeal or nasal swab was tested for SARS-CoV-2 by real time polymerase chain reaction (RT-PCR). We further tested all cases by RT-
PCR on day $0,3,6,9,12$ and 14 . We repeated the RT-PCR after 24 hours of first negative test, and viral clearance was considered after two consecutive negative tests. Those with inconclusive RT-PCR for SARS-CoV-2 were re-tested. We documented history, physical examination laboratory investigations, and chest $X$-ray from the records. Laboratory investigations included complete blood count, liver function tests, kidney function tests, inflammatory markers (erythrocyte sediment reaction (ESR), C-reactive protein (CRP) and ferritin) and prothrombin time-international normalized ratio (PTINR). High-resolution computed tomography chest was done in children with severe disease. Severity of illness was classified as per Dong, et al. [4] in four groups, viz. asymptomatic, uncomplicated illness,moderate disease, severe pneumonia/severe illness. All cases were treated as per institutional protocol and their clinical course was analyzed. Discharge criteria used was normal body temperature for $>3$ days plus two negative results on RTPCR for SARS-CoV-2. This study was approved by the institutional ethics committee. Written consent was obtained from guardians of patients.

We took first negative RT-PCR, out of two consecutive negative tests, for deciding day of viral clearance. It was considered the number of days from symptom onset to the first negative sample defining the duration of clearance. 
Statistical analyses: Data were summarized in proportions and median (IQR) was computed for viral clearance days. Chi square test was used for association between viral clearance days, age, sex and severity of disease. Results were computed using SPSS 22.0 (trial version).

\section{RESULTS}

Eighty-five eligible cases were enrolled in the study, of which 82 were discharged after fulfilling discharge criteria and three children died during the study. History of contact with COVID-19 cases could be traced in 52 children, out of which $24(28.2 \%), 10(11.8 \%)$ and $18(21.2 \%)$ cases had contact with parents [father $(n=10)$, mother $(n=9)$ and both $(n=5)$ ], with family member other than parents (siblings and grandparents) and with COVID-19 cases other than family members, respectively. None of the children had no known medical co- morbidity.

All the three three children who died had surgical comorbidities. One patient had ruptured liver abscess with peritonitis, another had multiple laryngeal papillomatosis, and the third child had been recently operated for ileal atresia.

Of the 16(18.8\%) symptomatic children, 7(40.4\%) had severe symptoms and $4(25 \%$, all $>5$ years of age $)$ had moderate symptoms. Viral clearance distribution in terms of sex, age, and symptoms is shown in Table I. The median (IQR) duration of viral shedding was 7(5-10) days with range of 2 to 45 days. Almost all children (98.8\%) showed viral clearance in 15 days. None of the cases presented as pediatric multisystem inflammatory syndrome (PIMS) in present study.

Among asymptomatic cases $(n=69)$, we found leukocytosis $(>10,000 / \mathrm{mm} 3)$ in $14.5 \%(n=10)$, leucopenia $(<4000 / \mathrm{mm} 3)$ in $11.6 \%(n=8)$, lymphopenia $(<1100 /$ $\mathrm{mm} 3)$ in $29 \%(n=20), \mathrm{CRP}>6 \mathrm{mg} / \mathrm{dL}$ in $7.3 \%(n=5)$, and $\mathrm{ESR}>20 \mathrm{~mm} / \mathrm{hr}$ in $23.2 \% \quad(n=16)$ cases. Among

Table I Trend of Viral Clearance in Children With SARSCoV-2 Infection $(\boldsymbol{N}=\mathbf{8 5})$

\begin{tabular}{lrrc}
\hline Patient characterstics & \multicolumn{3}{c}{ Viral clearance period } \\
\cline { 2 - 4 } & $<7 d(n=43)$ & $8-14 d(n=36)$ & $>14 \mathrm{~d}(n=3)$ \\
\hline Age, $y$ & & & - \\
Upto 1 y $(n=7)$ & $2(28.6)$ & $5(71.4)$ & - \\
1 to $<5$ y $(n=15)$ & $6(40.0)$ & $8(53.3)$ & $1(6.7)$ \\
$5-10$ y $(n=31)$ & $19(61.3)$ & $11(35.5)$ & $1(3.2)$ \\
$>10$ y $(n=29)$ & $16(55.2)$ & $12(41.4)$ & $1(3.5)$ \\
Male sex & $32(66.7)$ & $14(29.2)$ & $2(4.1)$ \\
Asymptomatic & \\
\hline
\end{tabular}

$P>0.05$ for all comparisons except ${ }^{a} P=0.007$ for comparison of duration of viral clearance among symptomatic and asymptomatic children. All values in no. (\%). symptomatic cases $(n=16)$ we found lymphopenia in all (severe lymphopenia, ( $<5 \%$ of total leucocyte count) in $43.8 \%$ ), leukocytosis in $37.5 \%(n=6)$, leucopenia in $31.3 \%$ $(n=5), \mathrm{CRP}>6 \mathrm{mg} / \mathrm{dL}$ in $93.8 \%(n=15)$ and $\mathrm{ESR}>20 \mathrm{~mm} /$ $\mathrm{h}$ in $75 \%(n=12)$ cases. D-dimer was evaluated in all symptomatic cases, and was found high $(>400 \mathrm{ng} / \mathrm{mL})$ in $37.5 \%(n=6)$ cases.

There were significant $X$-ray chest findings in 11 $(68.8 \%)$ of symptomatic cases. Main $X$-ray findings were bilateral infiltrates $(37.5 \%)$ and consolidation (31.3\%). Computed tomography (CT) was not done as a routine for all study subjects but in three children with severe respiratory distress. All three cases showed typical multifocal, bilateral, peripheral ground glass opacities.

Three patients needed mechanical ventilation. Most of moderate to severe category patients were managed with supportive therapy, antibiotics, hydroxychloroquine and lopinavir/ritonavir as indicated. However, none of our patients received remdesivir or tocilozumab as both these drugs were not available at the time of study.

\section{DISCUSSION}

The present study shows that most SARS-CoV-2 positive children were asymptomatic and $60 \%$ of the cases had household contacts. A higher number $(75.6 \%)$ of household contact was reported in a recent systematic review [5], though, others have reported majority $(75 \%)$ without any known contacts [6].

Other authors have reported 9-28\% asymptomatic cases in SARS-CoV-2 positive children [6-8]. Our findings differ from the description of the disease in previous studies, where the major presentation was a respiratory illness of varying severity [3-10], but are similar to findings from a recent meta-analyses $[1,11]$. This could be because of difference in population distribution of disease or difference in admission criteria. In contrast to above studies, we noticed cases with anosmia ( $n=4,25 \%$ of symptomatic cases) and gastrointestinal features $(n=4$, $25 \%$ of symptomatic cases). Although anosmia has been reported frequently in adult COVID-19 cases, only few case reports were available for the pediatric population [12]. The laboratory features were similar to previous pediatric reports [13].

Chest $X$-ray was normal in all asymptomatic cases in our study while $19 \%$ of clinically asymptomatic children had radiological abnormalities in a recent meta-analysis [14]. In a recent systematic review, Kumar, et al. [14] reported bilateral ground glass opacities in $40 \%$ of symptomatic cases; we found similar findings in the three cases that underwent a CT scan.Death rate in our study was higher than reported previously from China $(2.3 \%)$ and 


\section{WHAT THIS STUDY ADDS?}

- Median duration for 50\% viral clearance was 7 days, and almost all patients had viral clearance within 14 days.

- Symptomatic cases required significantly longer time for viral clearance.

lesser than reported by WHO (4.4\%) and from Italy (7.2\%) [15-16]. A study from Italy showed a higher proportion (16\% of admitted) of COVID-19 children needing ICU care [17], though the admission criteria were different.

$\mathrm{Xu}$, et al. [18] found a median (IQR) duration of 15 (11.7-18) days for viral clearance in 85 adults [18]. We did not find any relation of age, sex, and symptoms to viral clearance of SARS-CoV-2, similar to previous studies [19]. However, we could not document the relationship of viral load and viral clearance as we did not measure the viral load.

Our study shows that majority of children with SARS$\mathrm{CoV}-2$ infection were asymptomatic, and viral clearance was seen in majority within 14 days. This data needs supplementation from other centers, but will be useful for deciding on further infection control and quarantine guidelines, especially after school re-opening.

Ethics clearance: Institutional ethics committee of SMS Medical College; No. 423/MC/2020 dated 27 June, 2020.

Contributors: MLG, RKG, RBS: concept and design; SG, JSM, PKS, DD: data collection, review of literature; SG, RKG, JSM, PKS, DD: drafting of manuscript, SG, DKG: data analysis, statistics and data interpretation. MLG, RBS, RKG, SG: intellectual input, critical revision and finalization of manuscript. All authors provided final approval of version to be published. Funding: None; Competing interest: None stated.

\section{REFERENCES}

1. Meena J, Yadav J, Saini L, Yadav A, Kumar J. Clinical features and outcome of SARS-CoV-2 infection in children: A systematic review and meta-analysis. Indian Pediatr. 2020; 57:820-26

2. Sarangi B, Reddy VS, Oswal JS, et al. Epidemiological and clinical characteristics of COVID-19 in Indian children in the initial phase of the pandemic. Indian Pediatr. 2020;57: 914-17.

3. Indian Council of Medical Research. Revised Strategy of COVID19 testing in India (version 3, dated 20.03.20). Assessed September 08, 2020. Available from: https:// icmr.nic.in/sites/default/files/uploaddocuments/2020-320covid19testv3.pdf/www.mohfw.gov.in

4. Dong Y, Mo X, Hu Y, et al. Epidemiological characteristics of 2143 pediatric patients with 2019 coronavirus disease in China. Pediatrics. 2020; e20200702.

5. Hoang A, Chorath K, Moreira A, et al. COVID-19 in 7780 pediatric patients: A systematic review [published online ahead of print, 2020 Jun 26]. E Clinical Medicine. 2020;
100433.

6. Agha R, Kojaoghlanian T, Avner JR. Initial observations of COVID-19 in US children. Hosp Pediatr. 2020;10:902-5.

7. Qiu H, Wu J,Hong L, Luo Y, Song Q, Chen D. Clinical and epidemiological features of 36 children with coronavirus disease 2019 (COVID-19) in Zhejiang, China: An observational cohort study. Lancet Infect Dis. 2020;20:689-96.

8. Jingjing H, Guo Y, Mao R, Zhang J. Proportion of asymptomatic coronavirus disease 2019: A systematic review and metaanalysis. J Med Virol. 2020;1-11.

9. LudvigssonJF. Systematic review of COVID-19 in children shows milder cases and a better prognosis than adults. Acta Paediatr. 2020;109:1088-95.

10. Yang X, Yu Y, Xu J, et al. Clinical course and outcomes of critically ill patients with SARS-CoV-2 pneumonia in Wuhan, China: A single-centered, retrospective observational study. Lancet Respir Med. 2020;8:475-81.

11. Zhang L, Peres T, Silva M, Camargos P. What we know so far about coronavirus disease 2019 in children: A metaanalysis of 551 laboratory-confirmed cases. Pediatr Pulmonol. 2020;55:2115-17.

12. Mak PQ, Chung KS, Wong JS, Shek CC, Kwan MY. Anosmia and ageusia: Not an uncommon presentation of COVID-19 infection in children and adolescents. Pediatr Infec Dis J. 2020;39:e199-e200.

13. Yasuhara J,Kuno T, Takagi H, Sumitomo N. Clinical characteristics of COVID 19 in children: A systematic review. Pediatr Pulmonol. 2020;1-11.

14. Kumar J, Meena J, Yadav A, Yadav J. Radiological findings of COVID-19 in children: A systematic review and meta-analysis. J Trop Pediat. 2020:fmaa045 [published online 21 July, 2020].

15. Wu Z, Mc Googan JM. Characteristics of and important lessons from the coronavirus disease 2019 (COVID-19) outbreak in China: Summary of a report of 72/ 314 cases from the Chinese center for disease control and prevention. JAMA. 2020;323:1239-42.

16. Onder G, Rezza G, Brusaferro S. Case-fatality rate and characteristics of patients dying in relation to COVID-19 in Italy. JAMA. 2020;323:1775-76.

17. Grasselli G, Pesenti A, Cecconi M. Critical care utilization for the COVID-19 outbreak in Lombardy, Italy: Early experience and forecast during an emergency response. JAMA. 2020;323:1545-6.

18. Xu K, Chen Y, Yuan J, et al. Factors associated with prolonged viral RNA shedding in patients with coronavirus disease 2019 (COVID-19). Clin Infect Dis.2020;71:799-06.

19. Zhang W, Du RH, Li B, et al. Molecular and serological investigation of 2019-nCoVinfected patients: Implication of multiple shedding routes. Emerg Microbes Infect. 2020; 9:1:386-9. 